\title{
Impacto do Programa Bolsa Família sobre a Frequência Escolar: o caso da agricultura familiar no Nordeste do Brasil ${ }^{1}$
}

\author{
Raul da Mota Silveira Melo \\ Gisléia Benini Duarte ${ }^{3}$
}

\begin{abstract}
Resumo: $\mathrm{O}$ objetivo deste trabalho é avaliar o impacto do programa de transferência de renda condicionada Bolsa Família sobre a frequência escolar de crianças e adolescentes de cinco a 14 anos na agricultura familiar dos estados de Pernambuco, Ceará, Sergipe e Paraíba. Nessa investigação, o trabalho faz uso de dados primários (pesquisa de campo) e dados secundários (PNAD, 2005) para obter estimativas de propensity score. Os resultados indicam que, de forma geral, o programa eleva a frequência escolar das referidas crianças no intervalo de 5,4 a 5,9 pontos percentuais. Contudo, há importantes diferenças quando se considera meninas e meninos separadamente, sendo o programa eficaz no primeiro caso e ineficaz no segundo. Ou seja, apesar da avaliação positiva para as meninas, não parece haver efeito do programa sobre a frequência escolar dos meninos, o que pode estar associado a diferenças de gênero nos custos de oportunidades do investimento em capital humano no meio rural.
\end{abstract}

Palavras-chave: agricultura familiar, educação, Bolsa Família.

Abstract: The main proposal of this study was to evaluate the impact of the Bolsa Familia conditioned public cash transfer program on the school presence among the children and adolescents from five to 14 years, in the Brazilian states of Pernambuco, Ceará, Sergipe and Paraiba. The work uses both primary and secondary data (PNAD, 2005) to build two different control groups used for propensity score estimative matching with children from families that received income from the federal program. For all studied groups the impact of the Bolsa Familia was positive, in other words, the results indicate that the

1 Os autores, únicos responsáveis por potenciais erros e imprecisões da investigação, agradecem a Yony Sampaio pela oportunidade de utilização das informações da pesquisa de campo.

2 E-mail: rau.silveira@uol.com.br

3 E-mail: gisleiaduarte@gmail.com 
program increases the school presence by 5,6 points. But the results still suggest there is difference between gender, with the program being effective for girls, but not for boys. This probably is related to the gender difference in the opportunity cost of human capital investment in Brazil rural northeast.

Key-words: family farm, education, Bolsa Família.

Classificação JEL: O40, R11.

\section{Introdução}

Com cerca de 14,7 milhões de habitantes, o que representa quase metade da população rural brasileira $(47,2 \%)$ e aproximadamente $8 \%$ da população total, de acordo com a mais recente Pesquisa Nacional por Amostra de Domicílio (PNAD 2006), o meio rural do Nordeste do Brasil apresenta inúmeras características que o distingue, desfavoravelmente, das demais sub-regiões em termos de desenvolvimento social. Entre elas, dificilmente se pode apontar uma de mais fácil identificação, de maior representatividade desse atraso e de maior poder de constrangimento em relação a avanços sociais futuros que os baixos níveis de escolaridade da população.

Ainda conforme dados da PNAD, em 2006, a sub-região do Nordeste apresentou uma média de 2,5 anos de estudos para a população com 25 anos ou mais, contra 3,5 anos no caso da população rural brasileira e 6,7 anos para todo o País, considerando-se o mesmo grupo etário. Ou seja, o atraso escolar da referida sub-região chega a mais de quatro anos de estudos quando se compara com o nível de escolaridade do País e a um ano quando se tem o meio rural como referência. Salienta-se que, em sintonia com as evidências de maior persistência intergeracional de educação na região Nordeste e no meio rural apontadas por Ferreira e Veloso (2003), esse atraso se ampliou nos últimos anos; em relação ao Brasil como um todo, a defasagem do meio rural nordestino era de 3,7 anos em 1997.

Certamente, as defasagens atuais refletem atrasos passados quanto à frequência escolar na referida sub-região. Com efeito, microdados da PNAD para o ano de 1995 apontam que, enquanto menos de $75 \%$ das crianças e adolescentes entre cinco e 14 anos frequentavam escola no meio rural nordestino, no meio metropolitano, o percentual já superava os $90 \%$.

É importante, também, considerar as especificidades envolvidas no investimento em capital humano das famílias do meio rural brasileiro, em particular daquelas da agricultura familiar. Conforme estudos de Abramovay et al. (1998) e Kassouf (2001) a respeito da formação educacional nesse ambiente específico, 
aos menos no passado recente, não parecia haver uma crença na importância da educação formal como condicionante produtivo importante. Kassouf (2001) destaca a importância da persistência intergeracional da informação e do conhecimento entre os agricultores familiares. Mais recentemente, Mello, Silvestro, Abramovay, Dorigon, Ferrari e Testa (2003), a partir de pesquisa de campo, confirmam que, para a agricultura familiar, tendem a ficar no campo os filhos menos escolarizados, resultado já obtido por Durston (1996) para a América Latina. Mais importante para os propósitos deste trabalho, Mello et al. (2003) também apresentam evidências que sugerem que as restrições de renda não parecem ser a explicação mais importante para o investimento em capital humano das famílias agrícolas. A partir de níveis de escolaridade de agricultores de famílias com diferentes níveis de renda, esses autores mostram que as restrições de recursos apenas aparecem como limitantes quando há a intenção de migração para o meio urbano.

Esse último ponto torna o ambiente da agricultura familiar nordestino duplamente interessante para a avaliação de políticas públicas de transferências condicionadas de renda, como o programa Bolsa Família. Primeiro, por se tratar de um meio de histórica defasagem escolar em relação ao resto do País, revelando elementos de persistência intergeracional; segundo, por não parecer claro que as restrições de renda operem como restrição importante para o investimento em capital humano para aquelas famílias que têm interesse em permanecer na atividade. Pretende-se, neste trabalho, lidar com esse desafio, visando avaliar o impacto do programa Bolsa Família sobre a frequência escolar das crianças e jovens de cinco a 14 anos na agricultura familiar no Nordeste.

Nesse sentido, a presente investigação parece um complemento fundamental às avaliações existentes a respeito do impacto do Bolsa Família sobre a frequência ou escolaridade das crianças de famílias beneficiadas com recursos do programa. Os resultados obtidos por Cavalieri (2003) e Cardoso e Souza (2004), em avaliações mais gerais para o País, indicam que, apesar de não conseguir diminuir o trabalho infantil, o programa é capaz de aumentar a frequência escolar dos beneficiários do Bolsa Família.

Além desta introdução, o presente trabalho foi estruturado em mais quatro seções. Na próxima, são fornecidas evidências a respeito do atraso escolar relativo do meio rural nordestino a partir de diferentes gerações, enfatizando-se a situação atual das crianças e jovens. Na terceira seção, são descritos os dados e a metodologia utilizada na investigação. Os resultados das estimativas do impacto do programa Bolsa Família sobre a frequência escolar são apresentados e discutidos na quarta seção. E, na quinta e última seção, são expostas as conclusões do trabalho. 
Impacto do Programa Bolsa Família sobre a Frequência Escolar: o caso da agricultura familiar no Nordeste do Brasil

\section{Escolaridade e frequência escolar no meio rural nordestino: uma breve caracterização do retardo sub-regional}

Nesta seção, são apresentadas evidências a respeito dos níveis de escolaridade e frequência escolar no meio rural do Nordeste, comparando-as, sempre que possível, com os dados das demais sub-regiões nordestinas e do meio rural do País. O objetivo é, rapidamente, caracterizar o atraso da sub-região nordestina quanto à formação de capital humano da população, fornecendo subsídios para a discussão dos resultados obtidos na seção seguinte.

O Gráfico 1 apresenta os níveis médios de anos de estudo por ano de nascimento para as populações dos meios rural, urbano e metropolitano do Nordeste, e para a população rural brasileira. Além de permitir a comparação dos níveis educacionais entre os diferentes meios para as diferentes gerações, a estratégia fornece subsídios para a compreensão da origem da defasagem escolar da referida região ${ }^{4}$.

Gráfico 1. Média de anos de estudos por ano de nascimento - 2005.

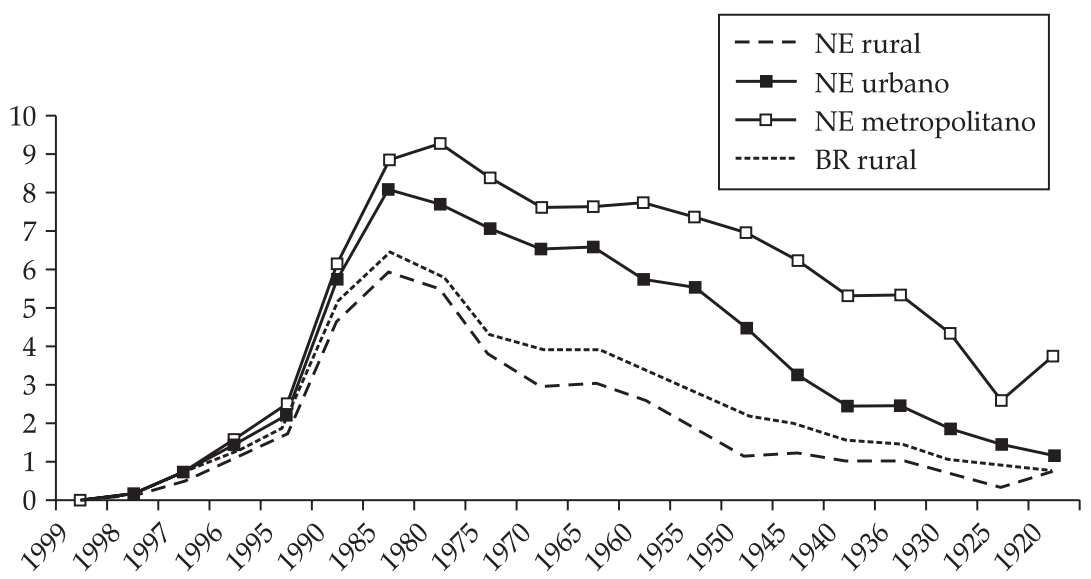

Fonte: Cálculos dos autores a partir dos microdados da PNAD-IBGE.

A partir das curvas do gráfico, nota-se que a defasagem escolar da população do meio rural nordestino vigora para todas as gerações e em relação a todas as demais populações dos outros meios. De fato, ainda que a representação

4 São considerados valores para o ano de 2005, em vez dos disponíveis para 2006, simplesmente pela consistência com os dados da pesquisa de campo utilizada no trabalho, que são referentes a 2005. 
permita aferir também o atraso geral do meio rural brasileiro, a situação do meio rural nordestino é ainda mais precária.

É possível perceber que essas diferenças inicialmente tendem a crescer, alcançando os valores mais elevados para a população nascida entre 1955 e 1970 - ou seja, para os indivíduos entre 35 e 55 anos -, e depois decrescer. Tal padrão é informativo, uma vez que reflete investimentos passados e políticas atuais de formação de capital humano nas diferentes sub-regiões consideradas. Assim, por razões distintas, as menores disparidades são encontradas para crianças e jovens e entre os mais idosos. No primeiro caso, muito provavelmente, o menor atraso da sub-região reflete a elevação das frequências escolares do período recente, como apontadas a seguir; no segundo caso, isto é, para a população mais idosa, as menores diferenças se devem à baixa escolaridade generalizada dos indivíduos mais idosos no País. Para as populações das faixas etárias intermediárias, que registram as maiores disparidades, o atraso do meio rural nordestino está associado à migração dos mais escolarizados para os meios urbanos $^{5}$ e, como se mostra adiante, às diferentes frequências escolares do passado recente encontradas entre as regiões.

De interesse mais imediato deste trabalho, vale ressaltar que, embora já menores, as defasagens de escolaridade hoje prevalecentes para as crianças do meio rural do Nordeste ainda são importantes e parecem condicionar de forma decisiva a progressão escolar futura. Buscando evidenciar esse fato, a Tabela 1 apresenta a média de anos de estudos das crianças e jovens de 6 a 14 anos, parte do universo foco da avaliação a seguir, dos quatro meios considerados anteriormente.

Tabela 1. Média de anos de estudos das crianças e jovens por idade - 2005.

\begin{tabular}{ccccc}
\hline Idade (anos) & NE Rural & NE Urbano & NE Metropolitano & Brasil Rural \\
\hline 6 & 0,0 & 0,0 & 0,0 & 0,0 \\
7 & 0,1 & 0,1 & 0,1 & 0,1 \\
8 & 0,5 & 0,7 & 0,7 & 0,6 \\
9 & 1,1 & 1,4 & 1,5 & 1,2 \\
10 & 1,7 & 2,2 & 2,4 & 1,9 \\
11 & 2,7 & 3,2 & 3,4 & 3,0 \\
12 & 3,4 & 3,8 & 4,2 & 3,8 \\
13 & 3,7 & 4,7 & 4,9 & 4,6 \\
14 & 4,4 & 5,3 & 5,6 & 5,3 \\
\hline
\end{tabular}

Fonte: Cálculos dos autores a partir de microdados da PNAD- IBGE.

5 Para uma discussão sobre as diferenças de escolaridade entre os originários do meio rural que migram e que não migram para área urbana, ver, por exemplo, Mello et al. (2003). 
Conforme a Tabela 1, além de estatisticamente significantes a partir dos oito anos de idade ${ }^{6}$, as defasagens escolares do meio rural nordestino em relação a todos os outros meios, em geral, crescem com o aumento da faixa etária. Comparando-se ao meio rural brasileiro, observa-se atraso de quase um ano de estudo para jovens de 14 anos e de apenas 0,2 ano para crianças de dez anos.

Diante da expansão recente do acesso escolar, tais evidências não são de fácil interpretação. Por um lado, podem refletir limitações ou mesmo ineficácia das políticas de incentivo à escolarização a partir de transferências condicionadas de renda (a exemplo do Bolsa Escola e do Bolsa Família); por outro lado, podem resultar apenas do elevado atraso inicial da sub-região nordestina. Em relação ao primeiro ponto, vale observar que o grande salto em termos de frequência escolar nos meios urbano e rural do Nordeste de 1995 a 2005 ocorre nos anos 90 (Gráfico 2).

Gráfico 2. Evolução da taxa de frequência escolar das crianças e jovens de 5 a 14 anos de idade.

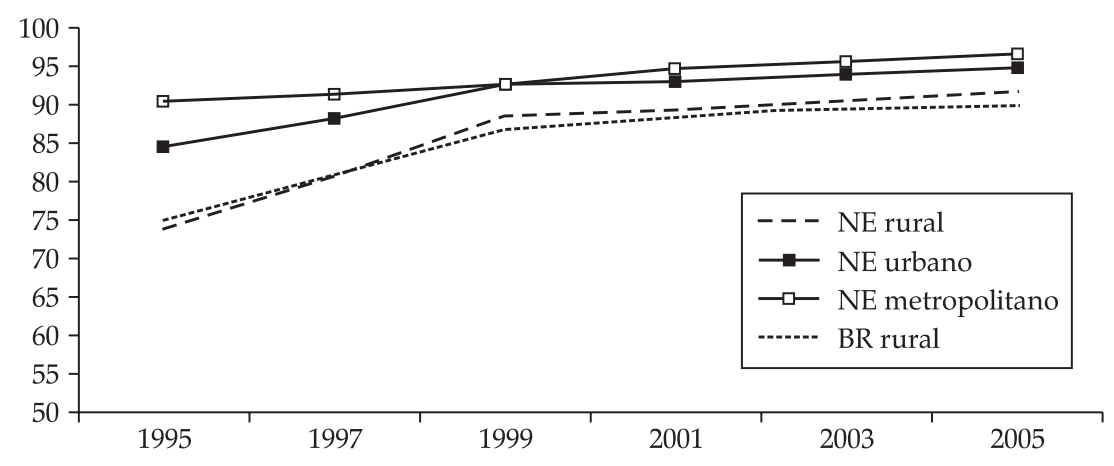

Fonte: Cálculos dos autores a partir dos microdados da PNAD-IBGE.

Conforme apresentado no Gráfico 2, as taxas de crescimento da frequência escolar para os meios rural e urbano do Nordeste e para o meio rural brasileiro são maiores entre 1995 e 1999 do que no período posterior, de 1999 a 2005, com um comportamento mais uniforme para crianças e jovens do meio metropolitano da região. Resultado desse maior ritmo de expansão, há clara tendência de convergência entre os meios urbano e rural do Nordeste no primeiro sub-período, o que aparentemente não acontece entre 1999 e 2005.

Para o período mais recente, tem-se que os níveis de disparidade entre as frequências escolares entre os meios rural e urbano ainda são relativamente elevados para o Nordeste e para o País. Em 2005, o percentual de crianças e jovens entre cinco e 14 anos que frequentavam escola no meio rural nordestino

6 Com significância estatística de 5\%, foram feitos testes para diferenças das médias para todos os anos. 
era de 91,6\%; no meio urbano, chegava a 94,6\% e no metropolitano, a 96,5\%, diferenças estatisticamente significantes a $1 \%$.

A despeito da criação do programa Bolsa Escola em 1999 e de sua incorporação e extensão por meio do Bolsa Família em 2003, não é evidente qualquer alteração significativa na trajetória de expansão da frequência escolar de crianças e jovens. Embora esse fato possa apenas retratar as dificuldades de expansão a níveis mais elevados de frequência escolar, dado o ainda significativo atraso do meio rural nordestino, parece imprescindível a avaliação das referidas políticas.

\section{Dados, características das amostras e metodologia}

Nesta seção, primeiramente, são descritas as fontes dos dados, a seleção das amostras e as características das famílias e crianças utilizadas nas estimativas do trabalho. Adicionalmente, discute-se a metodologia empregada para a obtenção dos resultados.

\subsection{Dados, seleção das amostras e características das famílias}

Dois conjuntos de informações foram utilizados neste estudo, um proveniente de pesquisa de campo (dados primários) e outro obtido diretamente da Pesquisa Nacional por Amostra de Domicílio (PNAD), ambos para o ano de 2005.

Os dados primários provêm da pesquisa de campo, do projeto Dom Helder Câmara (UFPE), realizada junto a famílias de produtores rurais dos estados de Pernambuco, Ceará, Paraíba e Sergipe. Essa amostra é composta por 745 crianças de domicílios que recebem ou não recursos do Bolsa Família. Essas famílias residem no meio rural e a atividade principal da propriedade é a agricultura. Desse conjunto, foram constituídos dois grupos, um formado por crianças e adolescentes de cinco a 14 anos que moravam em domicílios rurais beneficiados com recursos do Bolsa Família (grupo beneficiado pela política) e outro englobando domicílios rurais sem acesso a recursos do programa (grupo de controle 1).

A partir dos microdados da PNAD 2005, foi possível constituir um outro grupo de controle (grupo de controle 2), composto por crianças e jovens residentes em domicílios que não recebiam qualquer renda de transferência pública e que tinham o chefe trabalhando no meio rural em ocupação agrícola. Com o objetivo de restringir a referida amostra ao público alvo do Bolsa Família, adicionou-se com critério de inclusão nesse grupo a necessidade da renda familiar per capita ser menor ou igual a R\$ 200 mensais $^{7}$. Esse segundo grupo de controle é formado por 375 pessoas, de cinco a 14 anos.

7 Por precaução quanto ao tamanho da amostra, tal limite está um pouco acima do exigido para o Bolsa Família, que, em 2005, era R\$ 120,00 para renda familiar per capita. Os resultados obtidos neste trabalho não se modificam qualitativamente caso seja utilizada uma restrição de $\mathrm{R} \$ 150,00$. 
642 - Impacto do Programa Bolsa Família sobre a Frequência Escolar: o caso da agricultura familiar no Nordeste do Brasil

A Tabela 2 apresenta características pessoais, familiares e de localização das crianças e jovens das diferentes amostras utilizadas nas estimações do trabalho.

Tabela 2. Estatísticas descritivas das variáveis utilizadas.

\begin{tabular}{lcccc}
\hline \multicolumn{1}{c}{ Variáveis } & $\begin{array}{c}\text { Com Bolsa } \\
\text { Família - } \\
\text { campo }\end{array}$ & $\begin{array}{c}\text { Sem Bolsa } \\
\text { Família - } \\
\text { campo }\end{array}$ & $\begin{array}{c}\text { Geral - } \\
\text { campo }\end{array}$ & $\begin{array}{c}\text { Sem Bolsa } \\
\text { Família - } \\
\text { PNAD }\end{array}$ \\
\hline Características Pessoais & & & & \\
\hline Idade (anos, média) & 10,0 & 9,4 & 9,6 & 9,0 \\
Anos de estudo (anos, média) & 2,4 & 2,1 & 2,2 & 1,9 \\
Sexo: masculino (\%) & 51,8 & 42,8 & 44,9 & 51,0 \\
Cor : branca (\%) & 24,2 & 27,1 & 26,0 & 30,0 \\
Frequência escolar (\%) & 98,1 & 80,3 & 80,0 & 87,4 \\
\hline Características familiares & & & & \\
\hline Renda domiciliar per capita(R\$) & 43,1 & 49,8 & 47,2 & 44,1 \\
N. filhos 0 a 5 (\%) & 43,3 & 46,7 & 46,9 & 46,3 \\
N. filhos 6 a 15 (\%) & 97,1 & 92,8 & 94,0 & 94,3 \\
N. filhos mais 15 (\%) & 38,2 & 34,5 & 35,9 & 32,2 \\
N. membros & 6,8 & 5,9 & 2,4 & 5,1 \\
Escolaridade pai (anos, média) & 1,6 & 2,9 & 2,6 & 2,4 \\
Escolaridade mãe (anos, média) & 2,9 & 2,1 & 3,7 & 3,8 \\
Aposentadoria (\%) & 19,2 & 20,6 & 20,0 & 17,8 \\
\hline Localização & & & & \\
\hline Paraíba (\%) & 10,8 & 17,2 & 14,7 & 57,6 \\
Pernambuco(\%) & 26,6 & 41,4 & 35,3 & 42,3 \\
Sergipe (\%) & 44,5 & 19,4 & 29,2 & 16,0 \\
Ceará(\%) & 17,8 & 21,8 & 20,5 & 30,7 \\
\hline
\end{tabular}

Fonte: Pesquisa de campo e microdados da PNAD 2005.

Comparando-se a frequência escolar do grupo de tratamento com o controle da amostra de campo, nota-se que a frequência escolar é maior para o grupo de crianças que pertencem às famílias beneficiadas pelo Bolsa Família $(98,1 \%)$. As variáveis referentes à raça, idade e gênero não variam significativamente entre os grupos da amostra de campo e PNAD; em média, 72\% das crianças são de cor não branca, $51 \%$ são do sexo masculino, com nove anos de idade e têm dois anos de estudo.

Quanto às características familiares, a Tabela 2 indica que, em média, os pais dos domicílios beneficiados da amostra de campo apresentam escolaridade inferior à dos pais dos domicílios que não recebem Bolsa Família, da própria amostra. O contrário ocorre com a mãe; ou seja, em média, os domicílios beneficiados pelo programa contam com mães mais escolarizadas. As médias de anos de estudo dos pais para a totalidade da amostra de campo e para a PNAD rural são semelhantes. 
Para todos os grupos amostrais estudados, o percentual de famílias com filhos entre seis e 15 anos de idade é maior que o de famílias com filhos até cinco anos ou maiores de 15 anos. Em média, o grupo que recebe o Bolsa Família tem um número maior de membros. Por fim, a renda domiciliar per capita mensal também é semelhante entre os grupos, situando-se entre R 43 (beneficiários do Bolsa Família) e R \$4,80 (não beneficiários do Bolsa Família da pesquisa de campo). Tais valores indicam que, em média, as famílias consideradas estão dentro da faixa correspondente ao público-alvo do programa ( $\mathrm{R} \$ 120)$. Na amostra do presente estudo, a minoria das unidades familiares (menos de $20 \%$ ) possui algum membro que receba aposentadoria, um percentual que não varia significativamente entre as diferentes amostras.

Como o objetivo do trabalho é mensurar o impacto do programa Bolsa Família sobre a frequência escolar dos alunos que residem nos domicílios beneficiados, em função do possível viés de seleção envolvido da escolha das famílias beneficiadas, optou-se pelo método matching via propensity score, descrito na próxima seção.

\subsection{Estimativas do impacto do programa Bolsa Família sobre a frequência escolar: o matching via propensity score}

O impacto da aplicação dos recursos do Bolsa Família sobre a frequência escolar das crianças e jovens das famílias rurais beneficiadas pode ser visto dentro do problema geral de avaliação dos efeitos de políticas sociais ou públicas. Nesses casos, as dificuldades, de forma geral, derivam da impossibilidade da observação simultânea do indivíduo em situações ou estados diferentes, beneficiado e não beneficiado pela política. Ou seja, as técnicas tentam resolver o problema de avaliação sob insuficiência de informações a respeito dos beneficiados.

Para uma rápida formalização dessa situação, considera-se um indivíduo $i$, uma variável de avaliação de impacto $Y$ (frequência escolar, por exemplo) e os dois estados possíveis, "1" para a situação de ter sido beneficiado e " 0 " para a situação de não ter sido beneficiado. Com $D=1$ indicando o primeiro estado (ex.: família beneficiada pelo Bolsa Família) e $D=0$, o estado alternativo (ex.: não beneficiada pelo programa), o resultado para a variável de interesse da política, $Y$ do indivíduo $i$, pode ser representado por:

$$
Y^{i}=D Y_{1}^{i}+(1-D) Y_{0}^{1}
$$

o impacto da política para o indivíduo $i$ e o impacto médio da política sobre as famílias beneficiadas poderiam ser representados, respectivamente, por $\Delta^{i}=Y_{1}^{i}-Y_{0}^{i}$ e $\Delta=E\left(\Delta^{i} / D=1\right)=E\left(Y_{1}^{i}-Y_{0}^{i} / D=1\right)$, sendo $E\left(Y_{1}^{i}-Y_{0}^{i} / D=1\right)$ referente ao valor esperado condicionado à participação no financiamento.

Como não é possível observar as famílias/indivíduos nas duas situações, utiliza-se nas avaliações um grupo de indivíduos que não recebeu o benefício, 
644 - Impacto do Programa Bolsa Família sobre a Frequência Escolar: o caso da agricultura familiar no Nordeste do Brasil

grupo de controle, obtendo-se uma medida aproximada do impacto do benefício sobre a variável considerada:

$$
\begin{aligned}
& E\left(Y_{1}^{i} / D=1\right)-E\left(Y_{0}^{i} / D=0\right) \\
& =E\left(Y_{1}^{i} / D=1\right)-E\left(Y_{0}^{i} / D=1\right)+E\left(Y_{0}^{i} / D=1\right)-E\left(Y_{0}^{i} / D=0\right) \\
& =\Delta+E\left(Y_{0}^{i} / D=1\right)-E\left(Y_{0}^{i} / D=0\right)
\end{aligned}
$$

A última diferença do lado direito, entre os valores esperados da variável quando da não participação no programa condicionado aos dois estados, corresponde a uma medida do erro ao se utilizar o grupo de controle. Isto é, deriva do fato de que a taxa do aumento da frequência escolar, por exemplo, do grupo de controle não corresponde à dos beneficiados caso estes não tivessem recebido o Bolsa Família. Tal medida fornece, assim, um indicador do viés de seleção ou participação na política, ligado ao fato de que a participação no financiamento serve, em si, para diferenciar as famílias (mais motivadas versus menos motivadas, por exemplo), já condicionando os resultados do programa.

A precisão e o grau de identificação do impacto do programa sobre as famílias beneficiadas, $\Delta$, dependem, evidentemente, do tamanho do viés de seleção envolvido na avaliação. Essa magnitude, por sua vez, está vinculada ao mecanismo de seleção do grupo de controle e às técnicas de avaliação utilizadas na tarefa. A alternativa à inexistência de seleção aleatória entre beneficiados e não beneficiados, situação na qual não haveria qualquer viés de seleção ou participação, implica a utilização de grupo de controle escolhido de forma não aleatória, como é o caso do presente artigo.

Os métodos de matching por meio das estimativas de propensity score procuram sintetizar as informações contidas nas variáveis que afetam a participação no programa, através da estimação, condicionada nessas variáveis, da probabilidade de pertencer ao grupo de beneficiados (estimativa de propensity score $)^{8}$. Nesse sentido, assumem-se duas hipóteses fundamentais para garantia de uma estimação do impacto do programa livre do viés de seleção apontado. Primeiro, tem-se que, condicionando-se nas variáveis utilizadas na estimação do propensity score, não há diferença entre as probabilidades de participação no programa de um beneficiário e um não beneficiário. Segundo, assume-se que os resultados da variável de interesse $(Y)$, condicionados na probabilidade de participação no programa, são independentes da participação, ou seja, $\left(Y_{1}^{i}, Y_{0}^{i} \perp D_{i}\right) / \operatorname{Pr}\left(X_{i}\right)$, em que $\operatorname{Pr}\left(X_{i}\right)=\operatorname{Pr}\left(D=1 \mid X_{i}\right)$, sendo $\operatorname{Pr}\left(X_{i}\right)$ correspondente à probabilidade do indivíduo $i$ participar do programa e $X_{i}$, às variáveis que afetam a participação no programa; o símbolo $\perp$ denota independência estatística (ROSEMBAUM e RUBIN, 1983).

8 Para uma discussão mais técnica e detalhada do método ver, por exemplo, Angrist e Kruguer (1999) e Dehejia e Gatti (2002). 
Neste estudo, a estimação do propensity score foi feita por meio de um modelo logit, no qual a variável dependente é um indicador ou variável binária informando se a criança pertence a uma família que recebeu o benefício ou não e as variáveis de controle correspondem às características das crianças, da família e da localidade. Por sua vez, no sentido da aferir robustez dos resultados, o matching foi realizado a partir de três diferentes critérios: estratos, vizinho mais próximo (nearest neighbor matching) e uma função kernel.

O matching a partir de estratos ou grupos considera a comparação entre as médias da variável foco de interesse (ex.: frequência dos estudantes) dos beneficiados e não beneficiados pela política pública através estratos em que os indivíduos dos dois grupos apresentam, em média, a mesma estimativa de propensity score. O resultado final da avaliação do impacto da política é, então, dado pela média ponderada de cada estrato, com os pesos dados pela distribuição dos beneficiados em cada estrato.

Mais formalmente, considera-se a distribuição de estudantes beneficiados e não beneficiados pela política pública em $\mathrm{m}$ blocos ou estratos, de forma que a média das estimativas de propensity score para os dois grupos não apresente diferença estatisticamente significante em cada estrato. Se $Y$ é a variável de interesse, o primeiro passo é computar as diferenças de desempenhos entre os beneficiados e não beneficiados dentro de cada estrato:

$$
\Delta_{e}^{S}=\frac{\sum_{i \in S(e)} Y_{i}^{B}}{N_{e}^{B}}-\frac{\sum_{j \in S(e)} Y_{j}^{N B}}{N_{e}^{N B}}, \quad e=1,2, \ldots, m
$$

Em que $S(e)$ especifica o conjunto de indivíduos do estrato $e, Y_{i}^{B}$ e $Y_{j}^{N B}$ correspondemaos resultados observados para osindivíduos $i$ e $j$, respectivamente, dos grupos de estudantes beneficiados e não beneficiados (controle) no estrato, e $N_{e}^{B}$ e $N_{e}^{N B}$ referem-se aos respectivos números de indivíduos no mesmo estrato. Nesse caso, o resultado final da avaliação do impacto da política pública $\left(\Delta^{S}\right)$ é, então, computado a partir de uma média ponderada desses $m$ dados obtidos para os estratos:

$$
\Delta^{S}=\sum_{e=1}^{m} \Delta_{e}^{S} \frac{N_{e}^{B}}{N^{B}}
$$

Em que $N^{B}=\sum_{e=1}^{m} N_{e}^{B}$

Apesar de comparar indivíduos com médias de probabilidade de participação próximas em cada grupo, o matching a partir de estratos não garante a utilização de todas as observações disponíveis, uma vez que é possível que beneficiados ou indivíduos do grupo de comparação estejam ausentes em alguns estratos. O matching a partir do vizinho(s) com estimativa de propensity score mais próxima representa, pois, uma alternativa, já que parte da comparação de cada 
646 - Impacto do Programa Bolsa Família sobre a Frequência Escolar: o caso da agricultura familiar no Nordeste do Brasil

beneficiado pelo programa com o(s) indivíduo(s) do grupo de comparação (não beneficiado) com probabilidade de participação mais próxima, sendo o resultado final da avaliação do programa dado pela média dos resultados das comparações entre cada beneficiado. Com base nessas notações, $V(i)$, o conjunto de observações do grupo de comparação (não beneficiados) a ser relacionado ao beneficiado $i$, pode ser representado por:

$$
V(i)=\min _{j}\left\|p_{i}-p_{j}\right\|, \quad i \in B
$$

Sendo $p_{i}$ e $p_{j}$ correspondentes a probabilidades de ser beneficiado pelo programa e $\mathrm{B}$, ao conjunto dos beneficiados com a política pública.

Nota-se que a definição do(s) vizinho(s) a ser cotejado com cada observação do grupo de beneficiado (conjuntos do tipo $V(i)$ ) não obedece a nenhuma medida absoluta de distância em relação às estimativas de propensity score dos beneficiados, podendo haver comparação entre pares de observações.

O estimador de matching a partir de uma função densidade ou kernel matching representa, nesse sentido, uma ampliação do universo de comparação de cada estudante beneficiado, na medida em que estes são, de início, individualmente cotejados com todas as observações do grupo de controle (não beneficiados), ponderadas pelas distâncias de estimativas de propensity score através de uma função densidade. Novamente, o resultado final do impacto da política é obtido a partir de uma média das comparações feita entre os beneficiados. Mais formalmente, considerando-se uma função kernel $G$ e um parâmetro para janela $h$, para cada observação $i$ de estudante ou indivíduo beneficiado pela política pública (cada $i \in \mathrm{B}$ ), de início, é estimada a seguinte média ponderada dos resultados $Y_{j}^{N B}$ observados (considerando-se todos os estudantes ou indivíduos do grupo de controle, isto é, todo $j \in \mathrm{NB}$, grupo de não beneficiados):

$$
Y_{k i}^{N B}=\frac{\sum_{J \in N B} Y_{j}^{N B} G\left(\frac{p_{j}-p_{i}}{h}\right)}{\sum_{l \in N B} G\left(\frac{p_{j}-p_{i}}{h}\right)}, \quad i \in B .
$$

Finalmente, a estimativa do impacto da política $\left(\Delta^{K}\right)$ é obtida a partir da média das diferenças entre $Y_{i}^{B}$ e $Y_{k i}^{N B}$, isto é:

$$
\Delta^{K}=\frac{1}{N^{B}} \sum_{i \in B}\left[Y_{i}^{B}-Y_{k i}^{N B}\right]
$$

Deve estar claro, pois, que os estimadores que utilizam as estimativas de propensity score de fato permitem solucionar o problema da execução do balanceamento (match) entre estudantes quando o número de variáveis observáveis é muito elevado. Não obstante, não opera sem limitações; em particular, é sempre importante apontar que não soluciona problemas relacionados com o potencial viés de participação derivado da influência de variáveis não observáveis. 


\section{Estimativas do impacto do programa Bolsa Família sobre a frequência escolar na agricultura familiar do Nordeste}

As estimativas do impacto do programa Bolsa Família sobre a frequência escolar na agricultura familiar do Nordeste rural são levadas a efeito em duas etapas. $\mathrm{O}$ primeiro passo foi estimar a probabilidade das famílias receberem ou não o benefício, por meio do modelo logit. Nessa estimação, a variável dependente é uma dummy, que indica se a criança ou o jovem pertence a uma família que participa do programa de transferência de renda condicionada. Numa segunda etapa, tais dados são utilizados para comparar as taxas de frequência de crianças com probabilidades similares de pertencerem a domicílios beneficiados pelo Bolsa Família.

Tabela 3. Estimativas de modelo logit: impacto das variáveis sobre probabilidade de participação no programa Bolsa Família.

\begin{tabular}{lcccc}
\hline \multirow{2}{*}{ Variáveis } & \multicolumn{2}{c}{ Controle Campo } & \multicolumn{2}{c}{ Controle PNAD } \\
\cline { 2 - 5 } & Coeficientes (1) & Desvio padrão (2) & Coeficientes (3) & Desvio padrão (4) \\
\hline Sexo: Masculino & 0,129 & 0,098 & $-0,112$ & 0,108 \\
Cor: Branca & 0,013 & 0,119 & 0,214 & 0,132 \\
Idade & $0,049^{*}$ & 0,019 & $0,070^{* *}$ & 0,022 \\
Renda dom. per capita & $-0,001$ & 0,001 & $-0,005^{* *}$ & 0,001 \\
N filhos 0 a 5 & $-0,029$ & 0,063 & 0,022 & 0,067 \\
N. filhos 6 a 15 & $0,125^{* *}$ & 0,033 & $0,250^{* *}$ & 0,039 \\
N. filhos mais 15 & 0,002 & 0,046 & $-0,050$ & 0,054 \\
Escolar. Pai & $-0,034$ & 0,020 & $-0,011$ & 0,022 \\
Escolar. Mãe & $0,050^{*}$ & 0,016 & $0,038^{*}$ & 0,017 \\
Pai ausente & 0,074 & 0,215 & $-0,480^{*}$ & 0,204 \\
Ceará & 0,125 & 0,143 & $-0,193$ & 0,146 \\
Paraíba & 0,057 & 0,162 & 0,274 & 0,189 \\
Sergipe & $0,861^{* *}$ & 0,136 & $0,889^{* *}$ & 0,143 \\
Constante & $-1,491^{* *}$ & 0,255 & $-1,440^{* *}$ & 0,275 \\
N. observações & 745 & & 660 & \\
LR Chi2 & $90,09^{* *}$ & & $180,97^{*}$ & \\
\hline
\end{tabular}

* indica significância estatística a 5\%; ** indica significância estatística a 1\%. Nas colunas (1) e (2): 460 controles e 285 beneficiados. Nas colunas (3) e (4): 375 controles e 285 beneficiados.

Fonte: Estimativas dos autores a partir de dados de pesquisa de campo e PNAD.

Os resultados da primeira etapa (modelo logit) encontram-se na Tabela 3, com estimativas utilizando-se os dois grupos de controle (campo e PNAD). Dado o público-alvo do Bolsa Família, de forma geral, as estimativas estão dentro do esperado. Os valores da tabela indicam que as variáveis estatisticamente 
significativas foram a idade da criança, o número de filhos de seis a 15 anos, a escolaridade da mãe, a dummy de localização de Sergipe e a renda domiciliar per capita. Especificamente, os coeficientes indicam que famílias com filhos na faixa etária de seis a 15 anos têm uma maior chance de receber o benefício. Além disso, quanto mais escolarizadas são as mães, maior é a probabilidade dessas famílias se beneficiarem do programa. No caso do grupo de controle da PNAD, o coeficiente associado à variável renda domiciliar per capita indica que quanto maior a renda da família, menor a chance de participar do Bolsa Família.

Dado o menor número de variáveis estatisticamente significativas para o caso do grupo de controle da pesquisa de campo (colunas (1) e (2)), as estimativas da Tabela 3 também indicam um melhor balanceamento prévio entre beneficiados e indivíduos do referido grupo de controle (campo), em relação aos beneficiados e indivíduos do grupo de controle derivado da PNAD. Isso pode ser atribuído ao fato de apenas no primeiro caso serem cotejados indivíduos de mesmos estados e municípios. O melhor balanceamento prévio sugere, ainda, melhor potencial controle quanto às influências de características não observáveis na disposição das famílias em participar do programa.

Como indicado, as estimativas obtidas para os coeficientes do modelo logit permitem obter probabilidades estimadas dos grupos de beneficiados e controle, a partir das quais são feitos os pareamentos para obtenção do impacto do programa. No intuito de ilustrar e, ao mesmo tempo, evidenciar a importância do pareamento, os gráficos 3 e 4 apresentam as distribuições das probabilidades de participação no Bolsa Família estimadas para o grupo de beneficiados (em azul) e grupo de controle da pesquisa de campo (vermelho), respectivamente, sem e com o pareamento a partir do critério do vizinho mais próximo?.

Comparando-se as evidências dos referidos gráficos, numa situação de maior presença relativa de indivíduos beneficiados com as maiores probabilidades estimadas de participação no programa (Gráfico 3), o pareamento utilizado (Gráfico 4) permitiu relacionar crianças e jovens com probabilidades de participação bastante similares.

9 Em ambas as estimativas foi utilizado um kernel de Epanechnikov com janelas que minimizam o erro quadrado médio das estimativas. Os respectivos gráficos utilizando-se o grupo de controle da PNAD não são apresentados, mas podem ser disponibilizados pelos autores. 
Gráfico 3. Distribuições das probabilidades estimadas de participação no programa Bolsa Família para beneficiados e grupo de controle - sem pareamento.

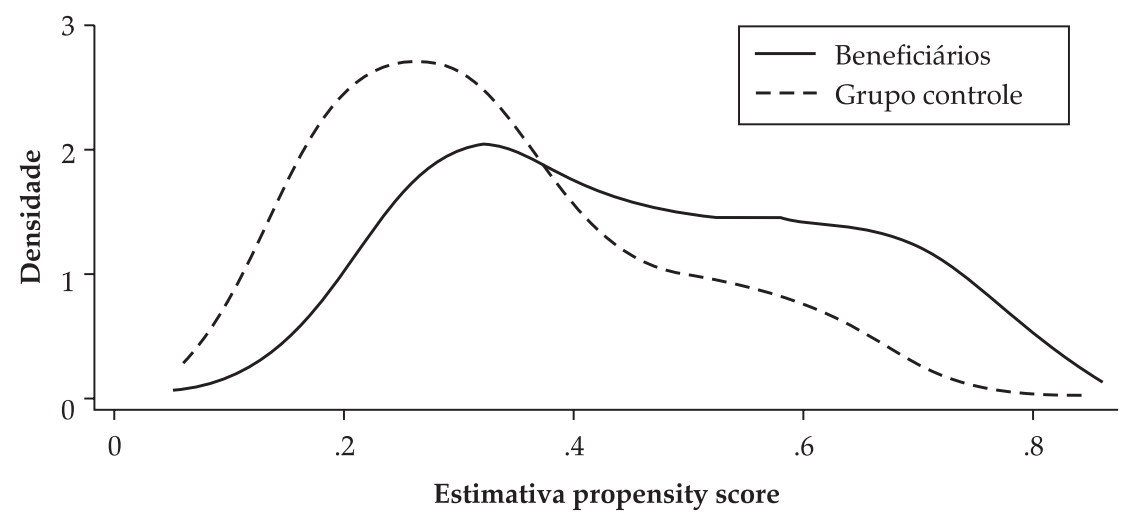

Fonte: Estimativas dos autores a partir de informações primárias.

Gráfico 4. Distribuições das probabilidades estimadas de participação no programa Bolsa Família para beneficiados e grupo de controle - com pareamento.

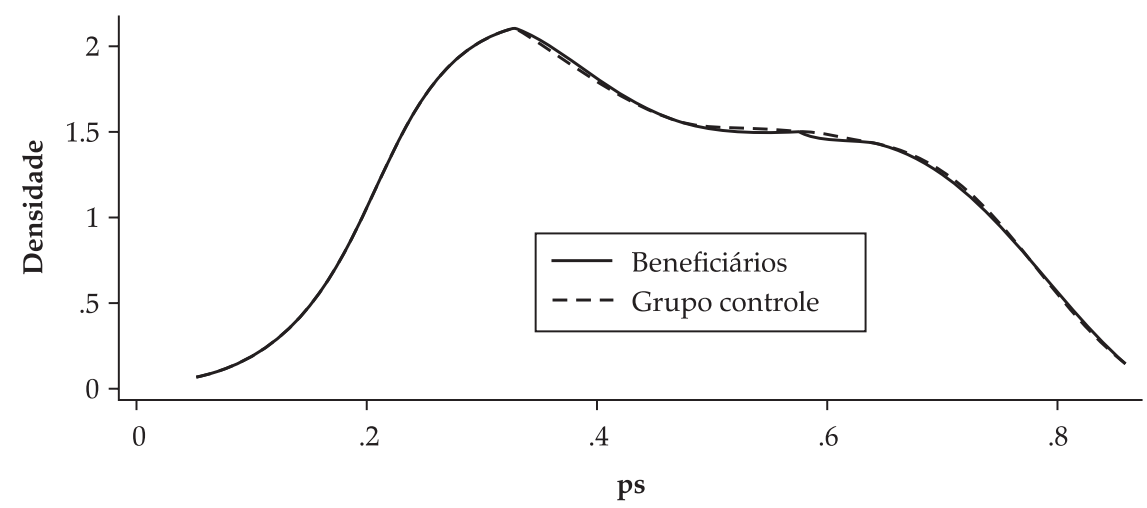

Fonte: Estimativas dos autores a partir de informações primárias.

Nas Tabelas 4 e 5, são apresentadas as estimativas do impacto do Bolsa Família sobre a frequência escolar das crianças e jovens de seis a 14 anos da agricultura familiar nos estados do Ceará, Paraíba, Pernambuco e Sergipe, obtidas tanto a partir do grupo de controle da pesquisa de campo como a partir do grupo de controle da PNAD. Nota-se que o conjunto de evidências aponta os resultados com e sem o pareamento ("Diferenças de médias) e que, na busca de maior robustez, são utilizados diferentes critérios de pareamento ou matching. 
650 - Impacto do Programa Bolsa Família sobre a Frequência Escolar: o caso da agricultura familiar no Nordeste do Brasil

Tabela 4. Estimativa do impacto do programa Bolsa Família sobre a frequência escolar Grupo de controle obtido através da pesquisa de campo - 2005.

\begin{tabular}{lccc}
\hline & $\begin{array}{c}\text { Frequência: } \\
\text { Beneficiários } \\
\mathbf{( 1 )}\end{array}$ & $\begin{array}{c}\text { Frequência: } \\
\text { Grupo de Controle } \\
\mathbf{( 2 )}\end{array}$ & $\begin{array}{c}\text { Impacto do } \\
\text { Bolsa Família } \\
(\mathbf{3})\end{array}$ \\
\hline Diferença entre médias & 0,982 & 0,878 & $0,104^{* *}$ \\
Matching via Propensity Score & $(0,008)$ & $(0,015)$ & $(0,017)$ \\
Vizinho mais Próximo & & & \\
Estratos & 0,982 & 0,926 & $0,056^{*}$ \\
& $(0,131)$ & $(0,262)$ & $(0,023)$ \\
& 0,982 & 0,878 & $0,054^{* *}$ \\
Kernel & $(0,008)$ & $(0,015)$ & $(0,015)$ \\
& & & \\
& 0,982 & 0,878 & $0,059^{* *}$ \\
& $(0,008)$ & $(0,015)$ & $(0,014)$ \\
\hline
\end{tabular}

* indica significância estatística a 5\%, ** indica significância estatística a 1\%. 460 controles, 285 beneficiados.

Fonte: Cálculo dos autores a partir de pesquisa de campo. Desvio padrão entre parênteses e obtidos por bootstrap para o caso do matching via propensity score. Na estimativa com matching via propensity score através de um kernel, foi utilizado o kernel de Epanechnikov.

Tabela 5. Estimativa do impacto do programa Bolsa Família sobre a frequência escolar Grupo de controle obtido a partir dos microdados da PNAD composto por indivíduos com renda domiciliar per capita abaixo de R\$ $200-2005$.

\begin{tabular}{lccc}
\hline & $\begin{array}{c}\text { Frequência: } \\
\text { Beneficiários } \\
(\mathbf{1})\end{array}$ & $\begin{array}{c}\text { Frequência: } \\
\text { Grupo de Controle } \\
(\mathbf{2})\end{array}$ & $\begin{array}{c}\text { Impacto do } \\
\text { Bolsa Família } \\
(\mathbf{3})\end{array}$ \\
\hline Diferença entre médias & 0,982 & 0,866 & $0,116^{* *}$ \\
Matching via Propensity Score & $(0,007)$ & $(0,017)$ & $(0,019)$ \\
Vizinho mais Próximo & & & \\
Estratos & 0,982 & 0,765 & $0,218^{*}$ \\
& $(0,131)$ & $(0,425)$ & $(0,089)$ \\
& 0,982 & 0,866 & $0,139^{* *}$ \\
Kernel & $(0,007)$ & $(0,017)$ & $(0,041)$ \\
& & & $0,149^{* *}$ \\
& 0,982 & 0,866 & $(0,037)$ \\
\hline
\end{tabular}

* indica significância estatística a 5\%, ** indica significância estatística a $1 \%$.

Fonte: Cálculo dos autores a partir de pesquisa de campo e microdados da PNAD. Desvio padrão entre parênteses e obtidos por bootstrap para o caso do matching via propensity score.. Na estimativa com matching via propensity score através de um kernel, foi utilizado o kernel de Epanechnikov.Controles 375, beneficiados 285.

As colunas (1) e (2) das tabelas 4 e 5 apresentam as médias das frequências, respectivamente, para beneficiários e não beneficiários do Bolsa Família. As 
colunas (3) das referidas tabelas mostram o impacto do programa (diferenças das médias) para os casos em que não se usa qualquer controle quanto à probabilidade de participação no Bolsa Família ("Diferenças entre médias"), utilizando-se estimativas de propensity score (Matching via Propensity Score).

$\mathrm{O}$ primeiro resultado geral a anotar é que, qualquer que seja o critério de matching e qualquer que seja o grupo de controle, as evidências indicam um impacto positivo do programa sobre a frequência escolar das crianças e jovens das famílias beneficiadas. As estimativas vão de um acréscimo de 5,4 (propensity score e controle campo, para a comparação a partir de estratos) a 21,8 pontos percentuais (propensity score e controle PNAD, para a comparação a partir do vizinho mais próximo), em média, na frequência escolar.

Dada a maior capacidade de controle para influências de características não observáveis com a utilização do grupo de controle de campo, os dados obtidos a partir desse grupo de comparação tendem a se aproximar do verdadeiro impacto do programa. Nesse sentido, nota-se, a partir da Tabela 5, que a não utilização das estimativas de propensity score levaria a uma sobre-estimação do impacto do programa sobre a frequência escolar (aumento de 10,4 pontos percentuais na frequência). Por outro lado, a sobre-estimação gerada com o grupo de controle derivado da PNAD indica que a incapacidade de controle para características municipais e locais dessa amostra pode ser um fator limitante ao uso das informações dessa fonte de dados em pesquisas de avaliação de impacto de políticas.

Outro ponto a ressaltar diz respeito aos resultados encontrados a partir de diferentes critérios de matching, por meio das estimativas de propensity score. Conforme apontado na Tabela 4, o impacto do programa situa-se entre 5,4 (pareamento ou matching a partir de estratos) e 5,9 pontos percentuais (pareamento ou matching a partir de uma função kernel), o que evidencia a robustez das estimativas.

É interessante apontar que os dados obtidos se assemelham aos apresentados por Cardoso e Souza (2004). Esses autores investigaram o impacto do Bolsa Escola sobre a frequência escolar e o trabalho infantil para meninos e meninas pertencentes a domicílios beneficiados ou não pelo Bolsa Escola para o ano de 2000. Os resultados indicam que o programa diminuía o tempo de trabalho da criança e aumentava a frequência escolar em cerca de três a cinco pontos percentuais.

Dadas as evidências apresentadas por Mello et al. (2003) a respeito das diferenças por gênero quanto ao investimento em capital humano das famílias agrícolas, também foram obtidas neste trabalho estimativas separadas para meninos e meninas. 
652 - Impacto do Programa Bolsa Família sobre a Frequência Escolar: o caso da agricultura familiar no Nordeste do Brasil

Tabela 6. Estimativa do Impacto do programa Bolsa Família sobre a frequência escolar Grupo de controle obtido através da pesquisa de campo - Filhas - 2005.

\begin{tabular}{lccc}
\hline & $\begin{array}{c}\text { Frequência: Bene- } \\
\text { ficiários (1) }\end{array}$ & $\begin{array}{c}\text { Frequência: Grupo } \\
\text { de Controle (2) }\end{array}$ & $\begin{array}{c}\text { Impacto do Bolsa } \\
\text { Família (3) }\end{array}$ \\
\hline Diferença entre médias & 0,979 & 0,873 & $0,106^{* *}$ \\
& $(0,012)$ & $(0,021)$ & $(0,023)$ \\
Matching via Propensity Score & & & \\
Vizinho mais Próximo & 0,979 & 0,897 & $0,082^{*}$ \\
Estratos & $(0,142)$ & $(0,305)$ & $(0,038)$ \\
& 0,979 & 0,873 & $0,079^{*}$ \\
Kernel & $(0,012)$ & $(0,021)$ & $(0,022)$ \\
& 0,979 & 0,873 & $0,089^{*}$ \\
\hline
\end{tabular}

* indica significância estatística a 5\%, ** indica significância estatística a 1\%. 260 controles, 146 beneficiados.

Fonte: Cálculo dos autores a partir de pesquisa de campo e microdados da PNAD. Desvio padrão entre parênteses e obtidos por bootstrap para o caso do matching via propensity score. Na estimativa com matching via propensity score através de um kernel, foi utilizado o kernel de Epanechnikov.

Tabela 7. Estimativa do Impacto do programa Bolsa Família sobre a frequência escolar Grupo de controle obtido através da pesquisa de campo - Filhos - 2005.

\begin{tabular}{lccc}
\hline & $\begin{array}{c}\text { Frequência: } \\
\text { Beneficiários } \\
(\mathbf{1})\end{array}$ & $\begin{array}{c}\text { Frequência: } \\
\text { Grupo de Controle } \\
\mathbf{( 2 )}\end{array}$ & $\begin{array}{c}\text { Impacto do } \\
\text { Bolsa Família } \\
\mathbf{( 3 )}\end{array}$ \\
\hline Diferença entre médias & 0,986 & 0,885 & $0,101^{* *}$ \\
& $(0,010)$ & $(0,023)$ & $(0,024)$ \\
Matching via Propensity Score & & & \\
Vizinho mais Próximo & 0,986 & 0,978 & 0,007 \\
Estratos & $(0,019)$ & $(0,146)$ & $(0,017)$ \\
& 0,986 & 0,885 & 0,030 \\
Kernel & $(0,010)$ & $(0,023)$ & $(0,023)$ \\
& 0,986 & 0,885 & 0,043 \\
& $(0,010)$ & $(0,023)-$ & $(0,018)$ \\
\hline
\end{tabular}

* indica significância estatística a 5\%, ** indica significância estatística a 1\%. 200 controles, 139 beneficiados.

Fonte: Cálculo dos autores a partir de pesquisa de campo e microdados da PNAD. Desvio padrão entre parênteses e obtidos por bootstrap para o caso do matching via propensity score. Na estimativa com matching via propensity score através de um kernel, foi utilizado o kernel de Epanechnikov.

Em relação às crianças e jovens do sexo feminino (Tabela 6), os valores indicam impactos positivos do programa, ainda mais significativos que os obtidos para a amostra com todos os gêneros, e confirmam a sobre-estimação do resultado derivado apenas da comparação das médias e a robustez das estimativas de propensity score. Para o grupo de controle da pesquisa de campo, a estimativa a partir do matching via propensity score aponta impacto positivo do programa entre 7,9 (matchinga a partir de estratos) e 8,2 pontos percentuais (matching a partir de uma função kernel). Ou seja, em média, o grupo de meninas das famílias beneficiadas pelo Bolsa Família apresenta uma proporção com 
frequência à escola superior entre 7,9 e 8,2 pontos percentuais em relação ao quadro em que não haveria transferência de renda pelo programa.

Diferente do registrado para as meninas, o impacto do Bolsa Família sobre a frequência escolar dos meninos (Tabela 7) só é positivo e estatisticamente significativo para o caso das diferenças das médias sem controle (diferença de 10,1 pontos percentuais). A estimativa do impacto do programa obtido através do matching via propensity score sob qualquer critério de matching não apresenta significância estatística. Ou seja, no caso dos meninos, o programa não se revela eficaz no sentido de aumentar a frequência escolar.

Embora o método de estimação utilizado não permita aferir as razões para as diferenças por gênero quanto ao impacto do programa Bolsa Família sobre a frequência escolar, o fato de se tratar de uma amostra de agricultores familiares sugere potenciais explicações. Nesse sentido, as diferenças apontadas anteriormente são consistentes com um custo de oportunidade para o estudo formal maior para os meninos que para as meninas. Segundo Cavalieri (2003), o filho se engaja mais cedo na atividade produtiva, quando comparado à filha, e a dedicação aos estudos implica menor tempo disponível para a atividade produtiva desenvolvida na propriedade.

Sob essa mesma perspectiva, Silvestro et al. (2001), quando analisam a relação entre educação e gênero na agricultura familiar, percebem que é entre os rapazes que se concentra a maior parte dos filhos que não tiveram acesso à educação formal. Esses autores sugerem que é o menor interesse das moças em permanecer no meio rural que faz com que elas busquem no ensino a oportunidade de inserção mais vantajosa nos meios urbanos.

Por fim, observa-se que as diferenças entre gêneros quanto ao impacto do Bolsa Família sobre a frequência escolar encontrada neste trabalho para universo da agricultura familiar do Nordeste do Brasil não estão presentes em Cardoso e Souza (2004), que analisam o País como um todo, embora também empreguem metodologias semelhantes. Tal constatação evidencia a importância de se considerar avaliações específicas segundo os diferentes ambientes sociais e econômicos.

\section{Conclusões}

O meio rural do Nordeste brasileiro apresenta inúmeras características que o distingue, desfavoravelmente, das demais sub-regiões do País em termos de desenvolvimento social. Entre elas destaca-se seu atraso quanto à formação do capital humano. Nesse meio, há evidência de que os agricultores familiares em particular têm baixo histórico de investimento na aquisição de escolaridade ou capital, uma situação aparentemente não relacionada diretamente com restrições de renda. Tais circunstâncias tornam o ambiente da agricultura familiar nordestino duplamente interessante para a avaliação de políticas públicas de transferências condicionadas de renda, como o Bolsa Família. 
654 - Impacto do Programa Bolsa Família sobre a Frequência Escolar: o caso da agricultura familiar no Nordeste do Brasil

Este artigo avaliou, para o ano de 2005, o impacto do programa de transferência de renda condicionada Bolsa Família sobre a frequência escolar entre crianças e adolescentes de cinco a 14 anos, para os estados de Pernambuco, Ceará, Sergipe e Paraíba. Para tanto, foram utilizados dados primários e secundários.

Os principais resultados, obtidos a partir de matching via estimativas de propensity score, indicam que, de forma geral, o programa aumenta a frequência escolar no intervalo de 5,4 a 5,9 pontos percentuais. Esse resultado é consistente com as evidências disponíveis fornecidas por Cardoso e Souza (2004) para o Brasil com um todo. Entretanto, diferentemente desses autores, os dados obtidos neste trabalho indicam importante diferença entre gêneros quanto ao impacto do programa Bolsa Família sobre a frequência escolar das crianças e jovens ligados à agricultura familiar do Nordeste. Observa-se efeito positivo apenas para indivíduos do sexo feminino, sem qualquer eficácia para aqueles do sexo masculino.

Por um lado, essa informação é consistente com as evidências apresentadas por Mello et al. (2003) a respeito do papel da renda familiar como condicionante ao investimento em capital humano nesse meio. Como sugerem esses autores, tal renda só parece ser um limitante para o avanço escolar das crianças ou jovens se o objetivo é a migração. Por outro lado, tendo em vista o ambiente específico da agricultura familiar, os resultados também podem estar de acordo com diferentes custos de oportunidades envolvidos na formação escolar das crianças e jovens de diferentes sexos.

Nessa perspectiva e num ambiente de baixa crença na importância da escolarização para desempenho das tarefas produtivas, é possível que os valores das transferências de renda do Bolsa Família sejam insuficientes para diminuir o tempo gasto com trabalho das crianças e jovens do sexo masculino na agricultura familiar do meio rural nordestino. Dada a importância do jovem do sexo masculino para as tarefas agrícolas nesse ambiente, é, além disso, duvidoso se a elevação das transferências permitiria a dedicação adequada desses jovens aos estudos. Assim, os resultados desta pesquisa sugerem que a política pública para quebra da persistência intergeracional da pobreza educacional no meio rural do Nordeste não deveria deter-se exclusivamente nos programas de transferências condicionadas de renda. Nesse ambiente, é necessário investigar se políticas adicionais que promovam a introdução de novas tecnologias, mais intensivas em capital humano, e a maior integração ao mercado não podem ser auxílios decisivos no desenvolvimento social e econômico desse meio.

\section{Referências Bibliográficas}

ABRAMOVAY, R.; SILVESTRO, M. L.; CORTINA, N.; BALDISSERA, I. T.; FERRARI, D. L.; TESTA, V. M. "Juventude e agricultura familiar: desafios dos novos padrões sucessórios". Brasília: Edições UNESCO, 1998. 101 p. 
ANGRIST, E.J. and KRUEGER, A.B. Empirical Strategies in Labor Markets. In. ASHENFELTER, O. and CARD, D., Handbook of Labor Economics, Vol. 3, NorthHolland, 1999.

CARDOSO, E., and SOUZA, A., P., (2004). The Impact Of Cash Transfers In Child Labor And School Attendance In Brazil. Working Paper No. 04-W07.Department Of Economics Vanderbilt University Nashville, Tn 37235.Www.Vanderbilt.Edu/Econ1

CAVALIERI, C., (2003). "Children's Contribution and Family Income: An Evaluation for Brazilian Rural Areas," mimeo. São Paulo: PUC.

DEHEJIA, R., and GATTI, R., 2002. "Child Labor: The Role of Income Variability and Access to Credit Across Countries," NBER Working Paper No. 9018, Cambridge

MA: National Bureau of Economic Research.

EMERSON, P. and SOUZA, A., (2002). "Bargaining over Sons and Daughters: Child Labor, School Attendance and Intra-Household Gender Bias in Brazil," Vanderbilt University, Dept. of Economics, Working Paper Series 02-w13.

FERREIRA, S.G. e VELOSO, F.A. "Mobilidade intergeracional de educação no Brasil", Pesquisa e Planejamento Econômico, V.33, N.3, dez. 2003, 481-513.

HECKMAN, J., H. ICHIMURA and P. TODD, 1997. "Matching as an Econometric Evaluation Estimator: Evidence from Evaluating a Job Training Program". Review of Economic Studies, 64.

KASSOUF, A. L. Trabalho Infantil. In: LISBOA, M.; MENEZES-FILHO, N.A., 2001, Microeconomia e Sociedade no Brasil. Rio de Janeiro, FGV.

RAVALLION, M., 2001. "The Mystery of Vanishing benefits: An Introduction to Impact Evaluation," World Bank Economic Review, 15(1), 115-140.

ROSENBAUM, P., and RUBIN, D., (1983). "The Central Role of the Propensity Score in Observational Studies for Causal Effects." Biometrika. Vol.70, pp.41-55.

SKOUFIAS, EMMANUEL and SUSAN PARKER, (2001). "Conditional Cash Transfers and Their Impact on Child Work and Schooling: Evidence from the Progresa Program in Mexico," Economía, 2 (1), pp.45-96.

MELLO, M., A.; SILVESTRO. M., L.; ABRAMOVAY, R.; CLOVIS DORIGON, C., (2003). Educação Formal e os desafios para a formação de uma nova geração de agricultores. XII Congresso da Sober - Sociedade Brasileira de Economia e Sociologia Rural.

ROSEMBAUM, R. and RUBIN, D. The Central Role of Propensity Score in Observational Studies for Causal Effects, Biometrika, Vol.70, 1983.

SILVESTRO, M. L.; ABRAMOVAY, R.; MELLO, M. A.; DORIGON, C.; BALDISSERA, I. T. Os impasses sociais da sucessão hereditária na agricultura familiar. Florianópolis/Brasília: Epagri/NEAD, 2001, 118 p. 